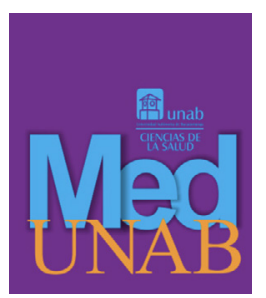

REVISTA DE LA FACULTAD

DE CIENCIAS DE LA SALUD

Vol. 24(2): 276-278, agosto-noviembre 2021

\title{
Secuelas del COVID-19, ¿qué viene ahora?
}

\section{Aftermath of COVID-19, what comes next?}

Rescaldo do COVID-19, o que vem a seguir?

\section{María Teresa Jáuregui-Buitrago, $M D ., \operatorname{Esp}^{1}$ iD}

1. Médica. Especialista en Epidemiología. Instituto de Seguridad del Trabajo, Chile.

Correspondencia. María Teresa Jáuregui Buitrago. Calle 4ta. Número 5-90 Pamplona, Norte de Santander. Colombia. Teléfono: +56948018087. Email: mjauregui166@unab.edu.co

\section{INFORMACIÓN DEL ARTÍCULO:}

Artículo recibido: 23 de diciembre del 2020

Artículo aceptado: 22 de junio del 2021

DOI: https://doi.org/10.29375/01237047.4063

Cómo citar: Jáuregui-Buitrago MT, Secuelas del COVID-19, ¿qué viene ahora?. MedUNAB. 2021;24(2):276-278 doi: https://doi.org/10.29375/01237047.4063

Palabras clave:

Infecciones por Coronavirus; Complicaciones; Fibrosis Pulmonar; Trombosis de la Vena; Rehabilitación.

Keywords:

Coronavirus Infections; Complications; Pulmonary Fibrosis; Venous Thrombosis; Rehabilitation.

Keywords:

Infecções por Coronavirus; Complicações; Fibrose Pulmonar; Trombose Venosa; Reabilitação.

\section{Señor editor.}

Desde que empezó la pandemia causada por el coronavirus COVID-19, hemos estado en un constante aprendizaje. Los países se han visto obligados a responder con sus mejores estrategias, para intentar que los sistemas de salud no colapsen y resguardar la vida de las poblaciones. Con este propósito, se han establecido medidas de contención y servicios de hospitalización organizados, además del papel fundamental de las unidades de cuidados intensivos para tratar las infecciones de gravedad, con lo cual se han evitado miles de muertes. 
A fecha de 11 de junio de 2021, en Colombia se habían reportado 3,724,705 casos, de los cuales $3,457,117$ corresponden a los recuperados, lo cual es asimilable a quienes no perdieron la vida. Sin embargo, desconocemos cuántos de ellos tendrán consecuencias físicas y emocionales derivadas de la infección por COVID-19 (1).

Aprendiendo de países que han tenido más tiempo para afrontar la pandemia y están estudiando las principales secuelas, encontramos que casi todos los sistemas del cuerpo humano pueden quedar afectados. El primero de ellos, dado el mecanismo de transmisión del virus, es el respiratorio. Se ha observado con frecuencia fibrosis pulmonar, muy importante por las implicaciones en cuanto a calidad de vida de los afectados, sobre todo por neumonía grave, y síndrome de distrés respiratorio del adulto (SDRA). Al principio se evidencia un patrón restrictivo en la espirometría, y disminución de la capacidad de difusión de monóxido de carbono. Los principales patrones histopatológicos son compatibles con daño alveolar difuso, neumonitis intersticial inespecífica y neumonía organizada.

En cuanto a la patogenia de su desarrollo es multifactorial y parcialmente conocida, es clara la relación con la enzima convertidora de angiotensina II, la tormenta de citoquinas, en especial interleucina 6, producción excesiva de radicales libres, con el consecuente aumento de la migración de células inflamatorias, que en muchos casos es tan intensa que genera más daños. A pesar de que la investigación aún está en curso, se ha podido evidenciar que la lesión pulmonar ocurre de forma más severa en hombres, mayores de 50 años, con antecedente de ingreso a UCI, hospitalización prolongada, uso de modos de ventilación no protectora, tabaquismo y reactantes de fase aguda elevados al ingreso (2).

Otras secuelas observadas en series de pacientes son: disnea persistente y dolor torácico, sin causa clara posterior a la enfermedad. No debemos olvidar que inherente a la estadía prolongada en UCI también se presentan lesiones inducidas por el ventilador y neumonías intrahospitalarias, que también requerirán atención neumológica y rehabilitación.

Por otra parte, en referencia a las secuelas de tipo cardiológico, se estima que cerca del $20 \%$ de pacientes con COVID-19 que requieren hospitalización presentan alguna manifestación cardiaca, aumento de troponinas, y mayor número de arritmias, situación que aumenta la mortalidad. Además, se ha descrito miocarditis viral en porcentajes variables, pero no despreciables. Se han probado en contra de los efectos deletéreos del virus la hidroxicloroquina a dosis bajas, por su papel en la remodelación del musculo cardiaco, y la inhibición de la metaloproteinasa de la matriz cardioespecífica (MMP9), además se ha estudiado la doxiciclina por su efecto similar en este sentido y así la posibilidad de revertir la disfunción del endotelio endocárdico. Otras sustancias que están aún en estudio incluyen la famotidina y los antioxidantes (3).

En un estudio de cohortes realizado en Alemania se pudo establecer que hasta el $60 \%$ de pacientes con antecedente de COVID-19 presentaban inflamación miocárdica independiente de sus comorbilidades, y el curso que haya tenido la enfermedad (4). Por estas razones, es preciso establecer una estrategia basada en un buen seguimiento de los pacientes, no descuidar las preexistencias, e implementar correctamente el uso de medicamentos cardioprotectores, como bloqueadores beta, y estatinas, con el objetivo de disminuir la carga de morbimortalidad que posiblemente aumente (5).

Los eventos trombóticos son igualmente frecuentes. Se ha descrito un aumento de marcadores como el dímero $\mathrm{D}$, fibrinógeno, tiempo parcial de tromboplastina activada, tiempo de protrombina, tendencia a trombocitosis leve $\mathrm{y}$ en pacientes que requieren hospitalización o UCI, un alto porcentaje de tromboembolismo venoso, por lo cual se han establecido múltiples esquemas terapéuticos con el fin de minimizar las complicaciones. En general, la heparina de bajo peso molecular es la más indicada en estos pacientes, y en quienes se presente trombocitopenia inducida por heparina, sería de elección el fondaparinux (6). Otras consecuencias incluyen síndromes postrombóticos, o la hipertensión pulmonar.

Ahora bien, desde el punto de vista neurológico, las afectaciones más frecuentes son leves: anosmia, que puede persistir semanas después de la recuperación del cuadro inicial; cefalea, confusión transitoria, y mialgias, también asimiladas por los pacientes como cansancio. El cuadro neurológico más prevalente en paciente con secuelas es el accidente cerebrovascular, reportado en estudios realizados en Wuhan (China), Reino Unido, y Nueva York (Estados Unidos). Particularmente se presenta en pacientes más jóvenes y aparentemente está relacionado con la coagulopatía, los altos niveles de fibrinógeno y dímero D (7). Más graves y afortunadamente más raras se han descrito encefalopatía necrotizante aguda, y el síndrome de Guillain Barre. En pacientes crónicamente enfermos, el empeoramiento de cuadros como el Parkinson y el deterioro cognitivo, se han relacionado con la susceptibilidad de células del hipocampo y el tronco encefálico para el coronavirus. 
Otras afectaciones muy comunes son las dermatológicas, entre las cuales se han incrementado las alergias, en especial en grupos como profesionales de la salud, y toda clase de dermatitis secundarias al uso de Equipos de Protección Individual (EPI). Otro efecto que genera impacto en la salud mental de los pacientes pese a su baja gravedad clínica es el efluvio telógeno, sobre todo en mujeres (8). Debido al uso de hidroxicloroquina a dosis altas, al inicio de la pandemia en países como China, se presentaron cuadros de hiperpigmentación, que casi siempre se revertían al descontinuar la sustancia.

Finalmente, en referencia a la afectación desde el punto de vista psiquiátrico, es común que gran número de personas presenten apatía, sensación de enlentecimiento, disminución de la percepción de bienestar e insomnio. En estudios en los cuales se evaluaron pacientes con infección confirmada por COVID-19, se describieron casos de depresión y trastorno de estrés postraumático, este último con una prevalencia muy alta en pacientes hospitalizados, además de empeoramiento de síntomas en patologías como los trastornos alimenticios y de ansiedad (9).

Dado el panorama, en nuestro país, múltiples programas de rehabilitación ya funcionan en la gran mayoría de clínicas y hospitales. Hay avances en cuanto al conocimiento científico. En abril del presente año se actualizó el consenso colombiano SARS-CoV-2 con todo lo referente a "Síndrome post COVID-19: complicaciones tardías y rehabilitación de los pacientes", avalado por las sociedades médicas colombianas (10). Así mismo, desde múltiples disciplinas que siempre han intervenido en el cuidado de los pacientes -fisioterapia, fonoaudiología, terapia ocupacional, psicología, entre otras-, se han generado lineamientos de atención, que incluso inician cuando el paciente aún se encuentra en la hospitalización, lo cual contribuye a disminuir el desacondicionamiento físico y emocional al que se enfrentan miles de personas y podrá contribuir de forma eficaz en un reintegro temprano a la vida y funcionalidad previa a la enfermedad. Hay que ver el futuro con esperanza, pero seguir estudiando y prepararnos para abordar de mejor manera lo que sigue.

Conflicto de intereses: La autora declara no tener conflicto de intereses.

\section{Referencias}

1. Instituto Nacional de Salud. COVID-19 en Colombia. Reporte 11-06-2021.7:45 pm. Bogotá, D.C. Recuperado a partir de https://www.ins.gov.co/ Noticias/paginas/coronavirus.aspx
2. Erico S, Todorovic A, Albuquerque A, Vieira E, Yuji $\mathrm{C}$, Valente M, et al. Fibrosis pulmonar secundaria a COVID-19: una revisión narrativa, [Internet] Experto Rev Respir Med. 2021 Abr 27; 1-13. Recuperado a partir de https://pubmed.ncbi.nlm.nih.gov/33902377/

3. Tyagi SC, Singh M. Daño multiorgánico por covid-19: insuficiencia cardíaca congestiva (cardiopulmonar) y fuga de la barrera hemato-cardíaca. Mol Cell Biochem. 2021;476(4):1891-1895. Recuperado a partir de https://www.ncbi.nlm.nih.gov/pmc/articles/ PMC7822399/

4. Puntmann VO, Carerj ML, Wieters I, Fahim M, Arendt C, Hoffmann J, et al. Outcomes of Cardiovascular Magnetic Resonance Imaging in Patients Recently Recovered From Coronavirus Disease 2019 (COVID-19). JAMA Cardiol [Internet]. 2020;5(11):1265-1273. doi: https://doi.org/10.1001/ jamacardio.2020.3557

5. Mitrani RD, Dabas N, Goldberger JJ. Lesión cardíaca COVID-19: implicaciones para la vigilancia a largo plazo y los resultados en los sobrevivientes. Ritmo cardíaco. 2020;17(11):1984-1990. https://doi. org/10.1016/j.hrthm.2020.06.026

6. Ruiz M. El impacto de la COVID-19 tras la enfermedad: los especialistas analizan sus secuelas [internet] 2020 Sept 29. Recuperado a partir de https:// economiadelasalud.com/topics/difusion/el-impactode-la-covid-19-tras-la-enfermedad-los-especialistasanalizan-sus-secuelas/

7. Vindegaard N, Benros ME. Consecuencias de la pandemia y la salud mental de COVID-19: revisión sistemática de la evidencia actual. Cerebro, comportamiento e inmunidad. 2020;89:531-542. https://doi.org/10.1016/j.bbi.2020.05.048

8. Saavedra Trujillo C. Sección IX. Síndrome post covid-19: complicaciones tardías y rehabilitación. Infectio. 2021;25(4):Suplemento 1. https://doi. org/10.22354/in.v25i4.979 